\title{
Comparative Activity of the Nitric Oxide (II) System the Process of Developing of An Immune Response in Guinea Pigs After Injection of Anti-Brucellosis Vaccines
}

\author{
Liliya A. Tukhvatullina1, Maxim A. Kosarev², Yana A. Bogova², Gulnara M. Safina², Ramil Y. \\ Nasibullin², Alfiya K. Gabbasova², Rufiya G. Karimova*1 \\ ${ }^{1}$ Kazan State Academy of Veterinary Medicine by N.E. Bauman, \\ Sibirsky tract 35, Kazan city, 420029, Russia \\ ${ }^{2}$ Federal Center for Toxicological, Radiation and Biological Safety, \\ Kazan city, Scientific City-2, 420075, Russia \\ Corresponding author email: Smolentsev82@mail.ru
}

\begin{abstract}
Despite the success achieved in the study of brucellosis, the problem of combating this infection remain relevant. There are a number of issues requiring a more profound research. Research work has been under way to find new and improve existing diagnostic methods, means of specific prophylaxis and methods of improving the farms with unfavorable brucellosis situation. The aim of our research was to perform a comparative study of immunogenesis in guinea pigs 15 , 30 and 60 days after their immunization with different cultures of strains: B. abortus 82-Rr, B. abortus 19, B. abortus R-1096 and their experimental infection with the vaccine strain B. abortus 82, and to determine the activity of the nitric oxide (II) system in guinea pigs bodies after vaccination. It was found that throughout the periods of research, guinea pigs vaccinated with cultures of B. abortus 82-Rr and R-1096 strains reacted negatively in serological reactions with a single brucellosis antigen, and positively in CBT (complement binding test) with R-antigen. In animals vaccinated with a strain 19 culture, positive indications of AR (agglutination reaction) and CBT with a single brucellosis antigen remained in high titers throughout the study periods, and were negative with R-antigen. A 100\% resistance to contamination with a culture of strain 82 was revealed in animals vaccinated with B. abortus 82-Rr and R-1096 strains, while in guinea pigs vaccinated with a vaccine made of strain 19 , a immunity breakthrough occurred and the immunogenicity was $60 \%$.
\end{abstract}

\section{KEY WORDS: BRUCELLOSIS, VACCINES, NITRIC OXIDE (NO), IMMUNOGENESIS, ANTIGENIC PROPERTIES.}

\section{INTRODUCTION}

Brucellosis is one of the most important zoonotic diseases in the world and is of particular importance in developing countries (Wang et al., 2021). This disease causes serious economic losses due to late abortions, stillborn and weak offspring. (Jing et al., 2020).

The Brucella family includes several independent species causing disease in various animal species, including

Biosc Biotech Res Comm P-ISSN: 0974-6455 E-ISSN: 2321-4007

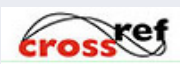

Identifiers and Pagination

Year: 2021 Vol: 14 No (9) Special Issue

Pages: $15-19$

This is an open access article under Creative

Commons License Attribn 4.0 Intl (CC-BY). DOI: http://dx.doi.org/10.21786/bbrc/14.9.4 humans. This family includes the following biological species: B. melitensis, B. abortus, B. suis, B. neotomae, B. ovis, B. canis, B. ceti, B. pinnipedialis and B. Microti. Detection of $B$. abortus in the cattle-breeding farm, or $B$. melitensis in the sheep-breeding farm and goat farms, $B$. suis at pig farm is the basis to regard the farm as unfavorable in terms brucellosis. (Ponomarenko et al., 2020). The source of the disease causative agent is animals sick with brucellosis. Infection of animals occurs by alimentary, sexual and aerogenic ways (Saeed et al., 2019). Clinical course of brucellosis in animals is characterized by polymorphism. The main symptom is abortion accompanied by massive and prolonged shedding of brucella with an aborted fetus, amniotic fluid, placenta, discharge from the genital and labor organs. Brucella is shedded by sick animals with urine and milk.
Article Information

Received: $13^{\text {th }} \mathrm{Apr} 2021$ ccepted after revision: $20^{\text {th }}$ June 2021 
The animals skin, stalls, litter, left-over food, care items, premises, as well as pastures and watering places are infected. Apart from abortions, brucellosis in animals can be accompanied by orchitis, bursitis, endometritis, mastitis. It can be in a latent form and be detected only by means of special tests. (Roberto et al., 2020). For the prevention of brucellosis in animals, vaccination is the only possible alternative to combat this infection. (Lalsiamthara and Lee, 2017). In spite of the fact there is still no perfect vaccine, vaccination with available vaccine strains remains the most successful method of preventing and controlling bovine brucellosis, and is an essential component of the majority of the brucellosis control and eradication programs worldwide (Onunkwo et al., 2018). The perfect vaccine should be both safe and immunogenic. General use of immunogenic vaccines will help reduce the incidence of brucellosis (Lai et al, 2017). Within the framework of a number of scientific studies there have been conducted experiments, noteworthy studies on the development of a bovine anti-brucellosis vaccine, the vaccination of which would provide for a sufficiently strong immunity without seropositivity (ElSayed et al., 2018).et al., 2018).

B. abortus 19 strain is a live attenuated vaccine and the first B. abortus vaccine widely used to combat brucellosis in cattle (Mohammadi and Golchin, 2018). The effectiveness of B. abortus S19 has been proven by experimental tests on cattle (Mascarenhas et al, 2020) and under field conditions (Mohamud et al., 2020). Its main characteristics are stable low pathogenicity, relatively high immunogenicity and moderate antigenicity. (Maruf et al., 2019). According to researchers, early serological studies 3-5 months after immunization with strain 82 vaccine contribute to a faster recovery of animals. (Ma Shu-Yi et al., 2020). The B. abortus82-Rr vaccine strain has a high-grade immunogenic activity, is mildly agglutinogenic, non-contagious, it meets the requirements for Brucella vaccine strains. 82-Rr strain differs from the original 82 strain by the ability to grow on growth media containing rifampicin in an amount of $50 \mathrm{\mu g} / \mathrm{ml}$ and the absence of abortogenic properties. Strain B. Abortus R-1096 is stable, non-contagious, nonagglutinogenic, has a fairly high-grade immunogenic activity, having no abortogenic properties (Saeed et al., 2019).

Nitric oxide (NO) is one of the main mediators of the immune system, as well as a participant of cell-to-cell cooperation. NO can have a damaging or protective effect on the cell functioning, depending on its concentration. Inducible NO synthase (i-NOS) hyperactivity produces damaging effect (Alhaji et al., 2016). Studies by Priscila Carneiro (2019) showed that the control of B. abortus replication in macrophages does not depend on the host cell NO, given the fact that infection of macrophages in the presence of NG-monomethyl-l-arginine, nitric oxide synthase does not affect the growth of intracellular bacteria. More important is the fact that the studies showed that B.abortus produces NO as a mechanism for regulating IL- $1 \beta$ secretion in macrophages, possibly through inflammation of NLRP3, as it was previously shown that the synthesis of this inflammasome is disrupted by the action of NO produced by B.abortus, which can be used by bacteria to modulate the secretion of IL-1 $\beta$ in infected mouse macrophages. Inflammasome NLRP3 is a protein complex being of crucial importance for the activation of caspase- 1 and maturation of neutrophil-activating factor IL-1 $\beta$ and IL-18 (Campos et al., 2019). The purpose of our research is to conduct a comparative study of the immunobiological properties of the most commonly used vaccines made of various $B$. abortus strains and to determine the activity of the nitric oxide (II) system in the body of guinea pigs after vaccination and contamination.

\section{MATERIAL AND METHODS}

The work was conducted in the laboratory of bacterial zooanthroponosis on guinea pigs with live weight of 400-450 g. They were divided into four groups. The first group was immunized subcutaneously with the B. abortus $82-\mathrm{Rr}$ vaccine in the left groin area with a dose of 1.5 billion m.c. by ITS (optical industry turbidity standard) in phosphate buffered saline in a volume of $1 \mathrm{ml}$. The second group was vaccinated with $B$. abortus 19 strain subcutaneously into the groin area with a dose of $1 \mathrm{bln}$ m.c. in a volume of $1 \mathrm{ml}$. The third group was vaccinated with the B. abortus R-1096 strain subcutaneously in the groin area with a dose of 1.5 bln m.c. in a volume of 1 $\mathrm{ml}$. The fourth group was left intact.

15 days, 1 and 2 months after vaccination, blood was taken from the ear vein of 3 animals from each group for serological tests. BPAT (buffered plate antigen test was conducted with brucellosis rose bengal antigen), AR, CBT, and CBT with R-antigen to the limiting antibody titers. 2 months after vaccination, guinea pigs were contaminated with the B. abortus 82 vaccine strain culture with a dose of $1 \mathrm{mln}$ m.c. subcutaneously into the right groin, opposite to the vaccine injection area, in a volume of 1 $\mathrm{ml}$. The strain culture was used as an infectious agent instead of the culture of the virulent Brucella strain. At that, bacteriological control of the infection dose was performed by means of inoculation of a suspension of brucella on Petri dishes with tryptosis agar.

Guinea pigs were euthanized a month after contamination, followed by serological and bacteriological tests. Bacteriological inoculations were conducted from 10 lymph nodes and organs. The inoculations were cultivated in a thermostat at $(37.0 \pm 0.5){ }^{\circ} \mathrm{C}$ for 15 days, viewing them 3 times after 5, 10 and 15 days. At the end of the cultivation of the inoculations, the infection index (II) of the guinea pigs bodies was determined by the culture of the vaccine strain using conventional bacteriological methods. Inoculated brucella cultures were typed in AR on glass with brucellosis S- and R-antisera.

The intensity of NO formation in the body was judged by the content in the blood serum of its conversion products - nitrite and nitrate anions, which were determined by reducing nitrates to nitrites with a single sample of zinc dust treated with an ammonia complex of copper sulfate 
with subsequent photometric determination of nitrites using the Griss reagent with a wavelength of $520.0 \mathrm{~nm}$ at $\mathrm{CPK}-3-01$. All results were statistically processed using Student's test.

\section{RESULTS AND DISCUSSION}

Serological studies of guinea pigs immunized with a B. abortus 82-Rr strain culture showed that not a single animal produced a positive result in any reaction with the S-antigen (BPAT, AR, CBT). Whereas CBT with $\mathrm{R}$-antigen is a sign of a strong immune response. Thus, on the 15th day after vaccination, the antibody titer in guinea pigs was $1.3 \pm 0.0 \mathrm{lg}$, on the 30th day they reached the highest titer, which was $1.7 \pm 0.12 \mathrm{lg}$, and on the 60th day it decreased to $1.3 \pm 0.0 \mathrm{lg}$. Table shows that guinea pigs vaccinated with a culture of strain 19, 15 days after immunization, reacted positively in BPAT, $\mathrm{AR}$ and CBT with S-antigen, CBT with $\mathrm{R}$ antigen was negative. On the 30th day, the titers of antibodies in AR and CBT with S-antigen, were $1.4 \pm 0.12 \mathrm{lg}$ respectively. The maximum titer of antibodies in CBT with S-antigen was $1.6 \pm 0.0 \mathrm{lg}$ on day 60 , and in AR $-1.3 \pm 0.0 \mathrm{lg}$, BPAT was also positive, while CBT with R-antigen - negative. The data of the third group indicate that not a single animal, in any reaction with the S-antigen, produced a positive result. While in CBT with $\mathrm{R}$-antigen reaction was with $1.8 \pm 0.0 \mathrm{lg}$ titre. One month after vaccination, the indicators of serological reactions with S-antigens, similar to the results after 15 days, were negative in all animals of this group.

Table 1. Indicators of antibody formation in guinea pigs vaccinated with various vaccines after 15,30 and 60 days

\begin{tabular}{|c|c|c|c|c|c|c|}
\hline \multirow[t]{3}{*}{ Strain culture } & \multirow{3}{*}{$\begin{array}{l}\text { Study } \\
\text { period }\end{array}$} & \multirow{3}{*}{$\begin{array}{l}\text { Number of } \\
\text { animals }\end{array}$} & \multirow[t]{3}{*}{ BPAT } & $\mathrm{AR}(\mathrm{lg})$ & \multicolumn{2}{|c|}{ CBT (lg) } \\
\hline & & & & \multicolumn{2}{|c|}{ Single brucellosis antigen } & R-antigen \\
\hline & & & & \multicolumn{3}{|c|}{$(\mathrm{M} \pm \mathrm{m})$} \\
\hline B. abortus $82-R r$ & \multirow[t]{3}{*}{15 days } & 3 & neg. & neg. & neg. & $1.3 \pm 0.0$ \\
\hline B. abortus 19 & & 3 & pos. & $1.7 \pm 0.24$ & $1.1 \pm 0.12$ & neg. \\
\hline B. abortus R-1096 & & 3 & neg. & neg. & neg. & $1.8 \pm 0.0$ \\
\hline B. abortus 82-Rr & \multirow[t]{3}{*}{30 days } & 3 & neg. & neg. & neg. & $1.7 \pm 0.12$ \\
\hline B. abortus 19 & & 3 & pos. & $1.4 \pm 0.12$ & $1.4 \pm 0.12$ & neg. \\
\hline B. abortus R-1096 & & 3 & neg. & neg. & neg. & $1.9 \pm 0.21$ \\
\hline B. abortus 82-Rr & \multirow[t]{3}{*}{60 days } & 3 & neg. & neg. & neg. & $1.3 .0 \pm 0.0$ \\
\hline B. abortus 19 & & 3 & pos. & $1.3 \pm 0.0$ & $1.6 \pm 0.0$ & neg. \\
\hline B. abortus R-1096 & & 3 & neg. & neg. & neg. & $1.7 \pm 0.12$ \\
\hline
\end{tabular}

In CBT with R-antigen, antibody titers increased - 93.3 \pm 35.9. And 2 months after vaccination, before infection, they decreased. The results of studies after contamination with the 82 strain culture are shown in table 2 . As it can be seen form the table, the antibody titers in AR with a single brucellosis antigen in two guinea pigs from the B. abortus 19 strain group, the lg index was $1.38 \pm$ 0.25 , whle in the rest AR and BPAT was negative. The highest titers in CBT with S-antigen were in guinea pigs vaccinated with B. abortus 19 strain culture - $1.84 \pm$ $0.13 \mathrm{lg}$, the lowest - in the control group was $1.6 \pm 0.0$ lg. In CBT with R-antigen, the highest titers were in the $B$. abortus 19 strain $-1.78 \pm 0.16 \mathrm{lg}$, and the lowest in B. abortus R-1096, $1.7 \pm 0.12 \mathrm{lg}$. In the group of animals vaccinated with the $B$. abortus 82-Rr strain, the antibody titers were $1.54 \pm 0.13 \mathrm{lg}$, and in the control group - 1.9 $\pm 0.0 \mathrm{lg}$, respectively.

The bacteriological study revealed that all animals of the control group had a high degree of inoculation of organs and lymph nodes with the contaminant culture, with II of $20.0 \pm 4.1$, with a spleen weight index of 2.0 \pm 0.47. Guinea pigs vaccinated with B. abortusR-1096 and B. abortus $82-\mathrm{Rr}$, the immune was 100\%. All animals resisted contamination with strain 82 culture. And in the group of animals vaccinated with the B. abortus 19 vaccine II was $16.0 \pm 13.6$, with SWI $1.68 \pm 0.14$ immunity was observed in $60 \%$ of the animals.
Figure 1. The level of nitrate- and nitrite-anions in the blood serum of guinea pigs vaccinated with B. abortus 82-Rr strain $\mu \mathrm{mol} / 1(\mathrm{n}=5)$

Note: ** - conclusively compared to the control group $(\mathrm{p}<0.001)$.

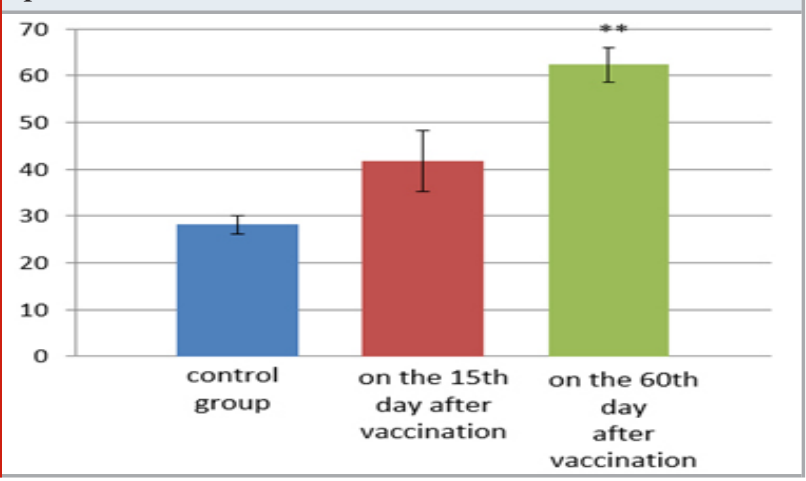

In vaccinated guinea pigs, the formation of nitric oxide (II) is more intense in comparison with the control group. Thus, in the blood of guinea pigs of the control group, the level of NO metabolites was $28.18 \mu \mathrm{mo} / \mathrm{l}$, while in the group of animals vaccinated with the B. abortus 82$\mathrm{Rr}$ vaccine on day 15, the level of NO metabolites was $41.81 \pm 1.9 \mu \mathrm{mol} / \mathrm{L}$, and on day $60-62.42 \pm 3.71 \mu \mathrm{mol} / \mathrm{l}$ $(\mathrm{P}<0,0001)$. Comparing the average NOx values of the guinea pigs vaccinated on day 15 and on day 60, we can conclude: on day 60 the NO activity is 1.49 times higher 
than on day 15 after vaccination (Fig. 1). The highest NOS activity after vaccination with the $\mathrm{B}$. abortus 19 strain is reached on day 60 being $37.87 \pm 3.04 \mu \mathrm{mol} / \mathrm{l}(\mathrm{p}<0.035)$, which is $9.5 \%$ lower than on day 15 (Fig. 2).

Figure 2: The level of nitrate- and nitrite-anions in the blood serum of guinea pigs vaccinated with B. abortus 19 strain $\mu \mathrm{mol} / \mathrm{l}(\mathrm{n}=5)$

Note: * - conclusively compared to the control group $(\mathrm{p}<0.01)$.

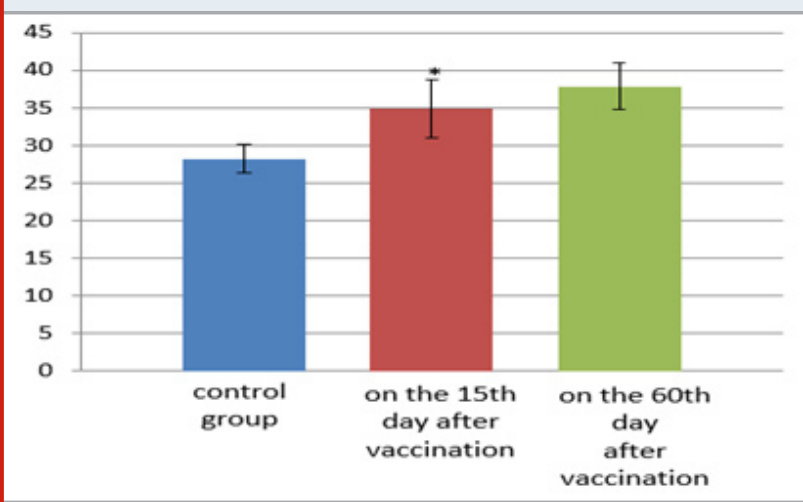

Figure 3: The level of nitrate- and nitrite-anions in the blood serum of guinea pigs vaccinated with R-109 strain $\mu \mathrm{mol} / \mathrm{l}(\mathrm{n}=5)$

Note: ** - conclusively compared to the control group $(\mathrm{p}<0.001)$.

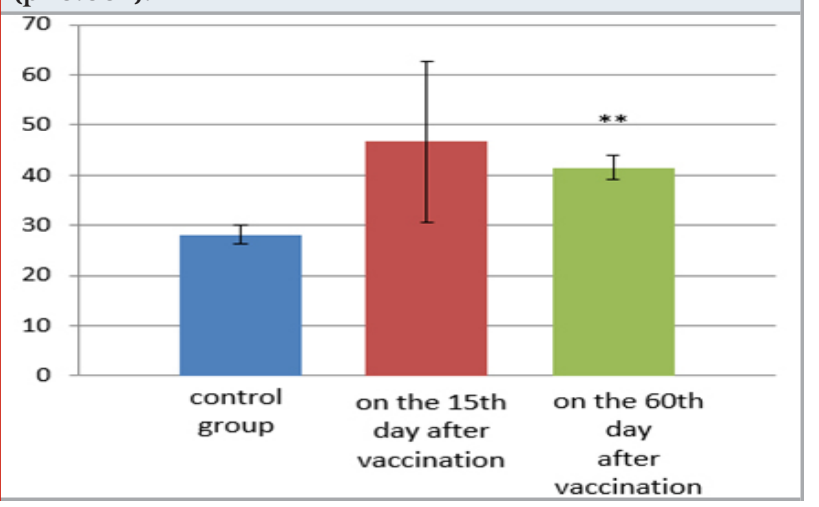

The level of stable nitric oxide metabolites of nitrite and nitrate anions on the 15 th day after vaccination with the R-1096 culture strain is $46.66 \pm 16.09 \mu \mathrm{mol} / \mathrm{l}$, and on the 60 th day $-41.51 \pm 2.43 \mu \mathrm{mol} / \mathrm{l}(\mathrm{p}<0.005)$. The highest content of active metabolites is on the 15th day after vaccination in comparison with the control group is is $65.57 \%$ higher, and in comparison with the group of vaccinated animals after 60 days - by $11.03 \%$ (Fig. 3).

The highest content of nitrite-initrate anions in blood serum in the group of animals vaccinated with the B. abortus 82-Rr vaccine on day 60 after vaccination was $62.42 \pm 3.71 \mu \mathrm{mol} / \mathrm{l}(\mathrm{p}<0.0001)$.

\section{CONCLUSION}

The work performed on guinea pigs to study the immunogenesis of various vaccine strains showed that during all research periods, cultures of B. abortus 82-Rr and R-1096 strains reacted negatively in all serological reactions with a single brucellosis antigen, and positively in CBT with R-antigen with high titers of antibodies. In animals vaccinated with a strain 19 vaccine, positive indications of AR and CBT with a single brucellosis antigen remained in high titers throughout the study periods, and were negative with R-antigen and only with an immunity breakthrough. A $100 \%$ resistance to contamination with a culture of strain 82 was revealed in animals vaccinated with B. abortus 82-Rr and R-1096 strains, while in guinea pigs vaccinated with a vaccine made of strain 19, a immunity breakthrough occurred and was 60\%.

We believe that the immune properties of anti-brucellosis vaccines can be checked using a culture of vaccine strain 82 as an infectious agent with a dose of $1 \mathrm{mln}$ m.c., but to make final conclusion, further research is needed to compare this method with the conventional one, when a culture of virulent strain B. abortus 54-M VGNKI (AllRussian State Center for Quality and Standardization of Medicines for Animals and Feed) is used for infection. The highest content of active metabolites is on the 60th day after vaccination of guinea pigs with the B. abortus $82-R r$ vaccine, and the lowest content of nitrite-nitrate anions is in the blood serum of the control group of animals. In vaccinated guinea pigs, the formation of nitric oxide (II) occurs more rapidly and the amount of antibody titer increases significantly. In view of the above, we can conclude that nitric oxide (II) is actively involved in the development of the immune response.

Source of funding: The authors declare that they have no funding support for this study.

Conflict of Interest: The authors declare that there is no known conflict of interest associated with this publication.

\section{REFERENCES}

Abdali, F., Hosseinzadeh, S., Berizi, E., Pourmontaseri, M. (2020). Prevalence of Brucella species in unpasteurized dairy products consumed in Shiraz province using PCR assay. Molecular biology research communications 9(3): 117-121.

Alhaji, N.B., Wungak, Y.S., Bertu, W.J. (2016). Serological survey of bovine brucellosis in Fulani nomadic cattle breeds (Bosindicus) of North-central Nigeria. Potential risk factors and zoonotic implications 153: 28-35.

Blasco, J., Moreno, E., Moriyon, I. (2016). Brucellosis vaccines and vaccine candidates. Veterinary Vaccines for Developing Countries 1-33.

Campos, P.C., Gomes, M.T.R., Marinho, F.A.V., Guimarães, E.S., de Moura Lodi Cruz M.G.F., Oliveira, S.C. (2019). Brucella abortus nitric oxide metabolite regulates inflammasome activation and IL- $1 \beta$ secretion in murine macrophages. European Journal of Immunology 49(7): 1023-1037. 
Daugaliyeva, 囚., Sultanov, A., Usserbayev, B., Baramova, S., Modesto, P., Adambayeva, A., Acutis, P., Peletto, S. (2018). Genotyping of Brucella melitensis and Brucella abortus strains in Kazakhstan using MLVA-15. Infection, genetics and evolution: journal of molecular epidemiology and evolutionary genetics in infectious diseases 58: 135-144.

El-Sayed, A., Awad, W. (2018). Brucellosis: Evolution and expected comeback. International journal of veterinary science and medicine 6:3 1-35.

Fomin, A,. Kosarev, M., Safina, G., Grigoryeva, S., Tukhvatullina, L. (2018). Titration of the optimal immunizing dose of B. abortus R-1096 strain in guinea pigs. Bulletin of Altai State Agrarian University 6 :164.

Goodwin, Z., Pascual, D. (2016). Brucellosis vaccines for livestock. Veterinary Immunology and Immunopathology 181: 51-58.

Gültekin, E., Uyanık, M.H., Albayrak, A. et al. (2021). Investigation of antibiotic susceptibilities of Brucella Strains isolated from various clinical samples in eastern Turkey. European Journal of Medical Research 26(1): 1-6.

Jing, H., Chao Pan, P.S., Erling, F., Jun, W., Li Z., Hengliang, W. (2020). Application of an O-Linked Glycosylation System in Yersinia enterocolitica Serotype 0:9 to Generate a New Candidate Vaccine against Brucella abortus. State Key Laboratory of Pathogen and Biosecurity, Beijing Institute of Biotechnology 8 (3): 436.

Kosarev, M.A., Fomin, A.M.,Safina,G.M., Bogova,Y.A., Tukhvatullina, L.A., Nasibullin, R.Y. and Karimova, R.G. (2021). Studying effective means and ways to specifically prevent brucellosis in small cattle. International Journal of Pharmaceutical Research 13(1): 3216-3224.

Lalsiamthara, J., Lee, J. (2017). Development and trial of vaccines against Brucella. Journal of Veterinary Science 18(1): 281-290.

Hull, N.C., Schumaker, B.A. (2018). Comparisons of brucellosis between human and veterinary medicine. Journal of Infection Ecology \& Epidemiology 8(1): $1-12$.

Lai, S., Zhou, H., Xiong, W. et al. (2017). Changing Epidemiology of Human Brucellosis, China, 1955-2014. Emerging infectious diseases 23(2): 184-194.

Ma Shu-Yi, Liu Zhi-Guo, Zhu Xiong; Zhao ZhongZhi,GuoZhi-Wei, Wang Miao, Cui Bu-Yun, Li Jun-Yan, Li Zhen-Jun. (2020). Molecular epidemiology of Brucella abortus strains from cattle in Inner Mongolia, China. Preventive veterinary medicine 183: 105080.

Mohammadi, E., Golchin, M. (2018). Detection of
Brucella abortus by immunofluorescence assayusing anti outer membrane protein of $19 \mathrm{kDa}$ antibody. Advances in Clinical and Experimental Medicine 27(5): 643-648.

Maruf, A.A., Yasmin, F., Yeasmin, F., Rahman, A.K.M.A., Hossain, M.M., Neubauer, H., Rahman, M.S. (2019). Comparison of humoral immune responses between heat-inactivated Brucella abortus biovar 3 and strain RB51 vaccines in indigenous cattle of Bangladesh. Journal of Veterinary Medical and One Health Research 1(2): 247-259.

Mascarenhas, D.R., Schwarz, D.G., Júnior, A.F., Oliveira, T.P. and Moreira, M.S.(2020).Validation of real-time PCR technique for detection of Mycobacterium bovis and Brucella abortus in bovine raw milk. Brazilian Journal of Microbiology 51(4): 2095-2100.

Mohamud, A.I., Mohamud, A.A., Rahman, M.S., Ehsan, M. A., Maruf, A. A.,Yasmin, F., Karim,F. and Neubauer, H. (2020). Comparison of humoral immune responses between cattle and buffaloes immunized with commercial brucella abortus strain rb51 vaccine in Bangladesh. J. Vet. Med 2(2): 405-415.

Onunkwo, J.I., Njoga, E.O., Njoga, U.J., Ezeokafor E.,Ekere, S.O. (2018). Brucella seropositivity in chicken and risk factors for Brucella infection at the animal-human interface in Anambra State, Nigeria. International Journal of One Health 4:28-34.

Ponomarenko, D.G., Rusanova, D.V., Khachaturova, A.A., Skudareva, O.N., Logvinenko, O.V., Rakitina, E.L., Kostyuchenko, M.V., Semenko, O.V., Maletskaya, 0.V, Kulichenko, A.N. (2020) Analysis of the epidemic and epizootic situation on brucellosis around the world in (2019) and the forecast for the Russian Federation for 2020. Problems of especially dangerous infections 2 : 48-56.

Roberto, F., Coloma-Rivero, Leonardo, G., Francisco, A., Waleska, S., Felipe, C., Sandra, C., Roberto, V. and Angel, A.0. (2020) The Role of the Flagellar Protein FlgJ in the Virulence of Brucellaabortus. In Cellular and Infection Microbiology;10: 178.

Saeed, U., Ali, S., Mahmood, Khan, T.M., Adawy, H. E., Melzer, F., Khan, A. U., Iftikhar, A., Neubauer, H. (2019). Seroepidemiology and the Molecular Detection of Animal Brucellosis in Punjab,Pakistan.Microorganisms 7(10) : 449.

Wang, Y., Xi,J., Wu, P., Zhang, H., Deng, X., Wang, Y., Ma, Z., Yi, J., Chen. C. (2021) Small ubiquitin-related modifier 2 affects the intracellular survival of Brucella abortus 2308 by regulating activation of the NF- $\otimes B$ pathway. Innate Immunity 27(1): 81-88. 